\title{
Interaction of fixation point and stimulus orientation on the appearance of rectilinearity*
}

\author{
LUBOMIR S. PRYTULAK† \\ University' of Western Ontario, London, Canada N6A $3 K 7$
}

\begin{abstract}
Data from two experiments indicate that the findings of Prytulak (1973) are generalizable to stimulus lines at all orientations: (a) when setting a response dot so that it appears to lie on an imaginary extension of a stimulus line, Ss misplace the dot on the side of the extension opposite the fixation point (a contralateral response, or CR) when the fixation point is in the vicinity of the stimulus line and on the same side of the extension as the fixation point (an ipsilateral response, or IR) when the fixation point is in the vicinity of the response dot; (b) CR is larger than IR; (c) fixation of the stimulus line or any point on its extension produces relatively accurate settings. New findings were: (a) the magnitude of CR decreased as the orientation of the stimulus line approached the vertical or horizontal; (b) the magnitude of CR on or near the horizontal was weaker than on or near the vertical; (c) the magnitude of IR was greater for stimulus lines lying in the upper half of the visual field than for stimulus lines lying in the lowerhalf. The difficulty of accounting for fixation point effects by means of physiological optics was discussed.
\end{abstract}

Prytulak (1973) had Ss set a response dot so that it appeared collinear with a stimulus line while fixating different parts of the stimulus display. The findings relevant here were that fixation of any point on the stimulus axis (which is the stimulus line and its imaginary extensions) produced accurate, that is collinear, set tings. Eccentric fixation (which is fixation off the stimulus axis), however, produced settings that were strongly and systematically noncollinear: during eccentric fixation in the vicinity of the stimulus line, $S$ placed the response dot on the side of the stimulus axis opposite the fixation point (a contralateral response, or CR); during eccentric fixation in the vicinity of the response dot, $\mathrm{S}$ placed the response dot on the same side of the stimulus axis as the fixation point (an ipsilateral response, or IR). Also, the degree to which S's setting deviated from collinearity was greater for CR than for IR.

In Prytulak (1973). lines at 45 and $315 \mathrm{deg}$ were tested ${ }^{1}$ but produced no significant main effect. But, as only two orientations were tested and as these were alike in being $45 \mathrm{deg}$ off the vertical, it is conceivable that orientation effects exist but were overlooked. The purpose of the two experiments reported below is to test stimulus lines at numerous orientations in order to determine whether the basic findings of CR and IR can be replicated at other orientations, and if they can, to determine whether their magnitude is affected by orientation.

\section{GENERAL METHOD}

The apparatus and procedure are detailed in Prytulak (1973). Briefly, $\mathrm{S}$ was presented with a stimulus card on which was a $1-\mathrm{cm}$ black stimulus line and a red-dot fixation point. On top of the stinulus card was an ace tate disk with a black response dot;

*This research was supported by Grant APA 390 from the National Research Council of Canada.

tThanks are due to Delbert $A$. Brodie for assistance in gathering the data.
$S$ could rotate the disk so as to move the response dot in a circle having a radius of $2.5 \mathrm{~cm}$ about one tip (the neartip) of the stimulus line. While fixating the red dot, $\mathrm{S}$ set the black response dot so that it appeared collinear with the stimulus line.

Occasionally, the fixation point might be indicated by instructions printed in red in the upper right-hand corner of the stimulus card, but no red dot (as. FIXATE NEARTIP or FIXATE RESPONSE DOT); when the fixation point was in the vicinity of the response dot, it was indicated by a red dot on the acetate disk rather than on the stimulus card.

The practice of describing the location of the fixation point by means of a rectangular coordinate system is continued below: one axis is the stimulus axis (and specifies elevation) and the other axis passes through the neartip (and specifies eccentricity), both in millimeters. The angle $\theta$ is the angle between the stimulus axis and an imaginary line between the neartip and the response dot.

\section{EXPERIMENT I}

\section{Method}

Nine male and 11 female students at the University of Western Ontario served as Ss.

Stimulus lines were presented at the eight orientations from 0 to $315 \mathrm{deg}$ in $45-\mathrm{deg}$ steps. The $\mathrm{S}$ was informed, by printed instructions, on which extension of the line to place the response dot: DOT LEFT or DOT RIGHT appeared with horizontal lines, and DOT ABOVE or DOT BELOW appeared with all others.

Six fixation points were tested at each orientation. Four of the fixation points had $0 \mathrm{~mm}$ eccentricity and were indicated by prina instructions but no red dot: FIXATE followed by MOVING DOT (the response dot), MIDDIST (the middle of the distance between neartip and response dot), TIP (the neartip), and MIDLINE (the middle of the stimulus line). The remaining two fixation points-at $\pm 5 \mathrm{~mm}$ eccentricity, $0 \mathrm{~mm}$ elevation-were marked by a red dot and accompanied by FIXATE RED DOT.

\section{Results}

Zero-eccentricity settings may be described merely as relatively accurate: $24 / 32$ of the mean $\theta$ s were smaller than $1 \mathrm{deg}$, and 30/32 were smaller than $2 \mathrm{deg}$. The two remaining mean $\theta$ s (along with the standard error of 

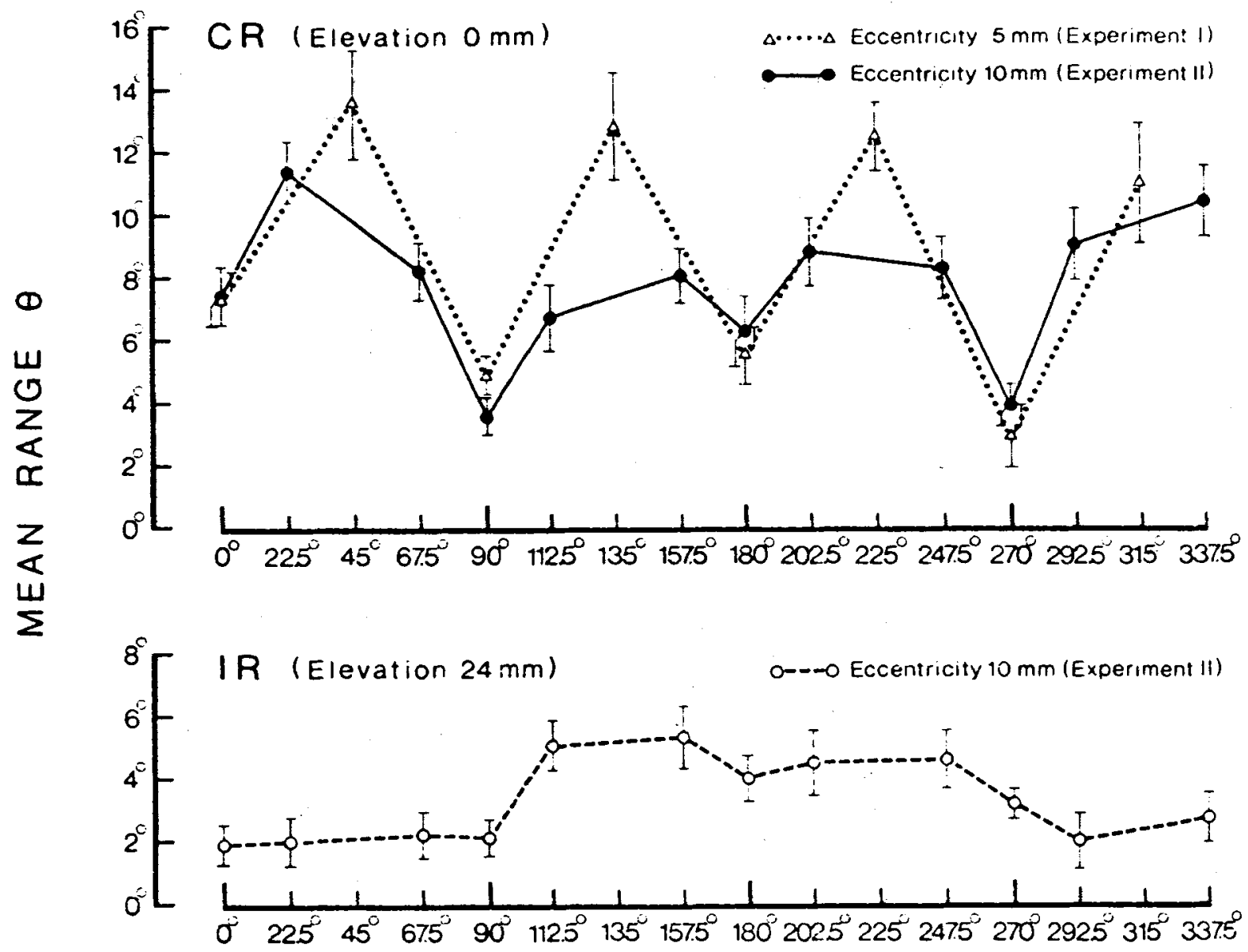

\section{ORIENTATION OF STIMULUS LINE}

Fig. 1. Mean range $\theta$ with \pm 1 standard error of each mean, Experiments I and $I 1$.

each mean) were settings above the stimulus axis during fixation of the 225 deg line: $2.67 \pm 0.93 \mathrm{deg}$ at $0 \mathrm{~mm}$ elevation (fixation of the NEARTIP) and $3.36 \pm 0.59 \mathrm{deg}$ at $12.5 \mathrm{~mm}$ elevation (fixation of the MIDDIST).

As for fixation at $\pm 5 \mathrm{~mm}$ eccentricity, $0 \mathrm{~mm}$ elevation, $16 / 16$ mean $\theta$ s were contralateral. The earlier finding of $C R$ during eccentric fixation in the vicinity of the neartip; then, appears to be generalizable to stimulus lines at numerous orientations.

Let us now turn to our chief interest--the magnitude of $\mathrm{CR}$ as a function of orientation. The dependent variable chosen to most succinctly convey the magnitude of the illusion experienced at different orientations is the difference between $\theta$ s resulting from fixation of corresponding points on opposite sides of the stimulus axis. Thus, for each stimulus line, the magnitude of CR was measured by computing the difference between $\theta \mathrm{s}$ produced by fixating $\pm 5 \mathrm{~mm}$ eccentricity at $0 \mathrm{~mm}$ elevation. This difference is referred to as range $\theta$.

The means of range $\theta$ (with \pm 1 standard error of each mean) are plotted in Fig. 1 as unfilled triangles within the dotted function. The other functions present data from Experiment II and will be discussed below Experiment I. Analysis of variance showed that orientation produced a significant effect, $F(7,133)=$ $16.62, p<.01$. Examination of the graphed data suggests the following regularities. First, horizontal stimuli (90 and $270 \mathrm{deg}$ ) produced slightly weaker effects than vertical stimuli ( 0 and $180 \mathrm{deg})$. Second, diagonal stimuli produced much stronger effects than vertical or horizontal stimuli: there is no overlap of standard error of the mean intervals between the four vertical-horizontal stimuli and the four diagonal ones.

\section{EXPERIMENT II}

\section{Method}

One male and 20 female students at the University of Western Ontario, as well as the present author, participated as Ss. Six of the females were also participants in Experiment $I$.

Stimulus lines were presented at 12 orientations- 4 on the 
vertical and horizontal and $822.5 \mathrm{deg}$ to either side of the vertical and horizontal. For each stimulus line. $\mathrm{S}$ fixated one of four red-dot fixation points. Two were drawn on the stimulus card, at $\pm 10 \mathrm{~mm}$ eccentricity and $0 \mathrm{~mm}$ elevation. The other two were on acetate circles, at $\pm 10 \mathrm{~mm}$ eccentricity and $24 \mathrm{~mm}$ elevation.

Instructions on which extension of the stimulus line to place the response dot were printed as in Experiment I. A second line of instructions read USE CIRCLE followed by A, B, or C. On Circle $A$ was a red-dot fixation point counterclockwise to the black response dot. On Circle B was a red-dot fixation point clockwise to the black response dot. Circle $C$ had only the black response dot and was used whenever $S$ was to fixate a stationary 0 -mm-elevation fixation point. Thus, $S$ was instructed to fixate the single red-dot fixation point that would be present at all times either on the card or on the circle.

\section{Results}

First, at $0 \mathrm{~mm}$ elevation, $24 / 24$ mean $\theta \mathrm{s}$ were contralateral. Experiment II, then, reinforces the finding of Experiment I, that eccentric fixation in the vicinity of the neartip produces $C R$ at all orientations.

Second, at $24 \mathrm{~mm}$ elevation, $24 / 24$ mean $\theta$ s were ipsilateral. It seems, then, that IR during eccentric fixation in the vicinity of the response dot is also replicable at all orientations. What about the magnitude of $\mathrm{CR}$ and IR?

A two-way analysis of variance of range $\theta$ showed three significant effects: a main effect of orientation $[F(11,231)=7.67, p<.01]$, a main effect of elevation $[F(1.21)=129.93, p<.01]$, and an Orientation by Elevation interaction $[F(11,231)=6.28, p<.01]$. As range $C R$ indicates settings opposite to range IR, the signs of the latter were reversed for the analysis. The means of range $\theta$ (with \pm 1 standard error of each mean) are plotted in Fig. 1: $0 \mathrm{~mm}$ elevation data are plotted as filled dots within the solid function, and $24 \mathrm{~mm}$ elevation data as unfilled dots within the dashed function. Let us consider the $0 \mathrm{~mm}$ elevation data first.

The underlying pattern seems to be as follows. As in Experiment I, horizontal stimuli (90 and $270 \mathrm{deg})$ produced slightly weaker effects than did vertical stimuli ( 0 and $180 \mathrm{deg}$ ). Also, of the two diagonal stimuli lying in any of the four quadrants, the one nearer the horizontal produced the weaker effect in $4 / 4$ comparisons. (In the quadrant bounded by 0 and $90 \mathrm{deg}$, for example, the effect at $67.5 \mathrm{deg}$ was smaller than at $22.5 \mathrm{deg}$.) Together, the observation from Experiment I and the two observations from Experiment II suggest that the appearance of stimuli on or near the horizontal is less affected by fixation point than the appearance of stimuli on or near the vertical.

Second, as in Experiment $I$, diagonal stimuli were more affected by fixation point than vertical or horizontal stimuli. Thus, the effect at any vertical or horizontal orientation was always weaker than the effect at the two diagonal orientations immediately clockwise to it, or the two immediately counterclockwise. Also, of the 12 means in the solid function, the 4 from vertical or horizontal stimuli produced the three lowest means and the fifth lowest-the single reversal being that the mean for the diagonal stimulus at 112.5 deg was lower than it was for the vertical stimulus at $0 \mathrm{deg}$. This small discrepancy can be attributed to a preponderance of the first regularity noted above over the second: in the first place, 112.5 deg should produce a weaker effect than 0 deg because it is near the horizontal; in the second place, it should produce a stronger effect because it is diagonal.

Third, the comparison of effects at inclinations of $45 \mathrm{deg}$ with effects at inclinations of $22.5 \mathrm{deg}$ is complicated by the former being taken from Experiment I, where eccentricity was $5 \mathrm{~mm}$, and the latter from Experiment II, where eccentricity was $10 \mathrm{~mm}$. The similarity in Experiments I and II of effects for stimuli at $0,90,180$, and $270 \mathrm{deg}$, however, suggests that the difference between experiments was slight and that the very large effects at $45 \mathrm{deg}$ will prove larger than those at $22.5 \mathrm{deg}$ when the comparison can be made within a single experiment and using a constant eccentricity. In Fig. 1 , it can be seen that in any quadrant, the effect at the 45-deg inclination is always stronger than the two effects at the 22.5-deg inclinations. Also, of the 12 diagonal stimuli from the two experiments, the 4 at $45-\mathrm{deg}$ inclinations produced the three largest and the fifth largest mean range $\theta$. Furthermore, as the stimulus $22.5 \mathrm{deg}$ off the vertical consistently produced a stronger effect than the stimulus in the same quadrant $22.5 \mathrm{deg}$ off the horizontal, one might expect that the very strongest effects will eventually be found for stimuli slightly less than $45 \mathrm{deg}$ away from the vertical.

Fourth, the magnitude of IR (dashed function, lower graph) is always weaker (12/12 comparisons) than the CR produced by a stimulus line at the same orientation. Also, IR seems less responsive to the orientation of the stimulus line-although a dip of the function at vertical and horizontal orientations can be discerned, the dip is shallow, and at $270 \mathrm{deg}$, equivocal. Nevertheless, an analysis of variance of the IR data alone shows that orientation did have a significant effect $[F(11,231)=$ $3.11, p<.01]$. Rather than viewing the IR effect as weakening toward the vertical and horizontal, however, one might be more taken with the regularity that each of the seven stimuli between 90 and 270 deg produced a stronger effect than any of the seven stimuli between 270 and $90 \mathrm{deg}$-which is to say that, at $24 \mathrm{~mm}$ elevation, displays in which the stimulus line lay in the upper half of the visual field produced stronger effects than did displays in which the stimulus line lay in the lower half of the visual field.

\section{DISCUSSION}

The research reported above attempts to contribute to an understanding of the nature of distortion in the peripheral visual field, but does not attempt to ascertain the origin of that distortion. It may be useful. 
nevertheless, to point out that the distortion is unlikely to originate in the optical portion of the visual system. Prytulak (1973) discussed how optics is able to account for CR but not for IR. Now, however, even its ability to account for $C R$ must be qualified-it cannot account for the effect of orientation on the magnitude of CR. To this writer's knowledge, physiological optics possesses no characteristic which would increase the distortion of lines increasingly tilted away from the vertical or horizontal.

The attribution of the orientation effects reported above to postoptical mechanisms is reinforced by the finding that at least one other orientation effect is independent of optics: Campbell, Kulikowski, and Levinson (1966) showed that the deteriorating resolution of a grating, as it rotates from the vertical or horizontal, persists even when the dioptrics of the eye are bypassed by using a neon-helium laser to form Thomas Young interference fringes directly on the retina. If this effect of orientation on resolution is known to be postoptical, then, perhaps it is less implausible to suppose that the orientation effect reported above is postoptical as well.

\section{REFERENCES}

Campbell, F. W., Kulikowski, J. J., \& Levinson, J. The effect of orientation on the visual resolution of gratings. Journal of Physiology, 1966, 187, 427-436.

Prytulak, L. S. The effect of fixation point on the appearance of rectilinearity. Perception \& Psychophysics, 1973, 14, 387-393.

\section{NOTE}

1. If the stimulus line is the arm of a clock (with the response dot always set at the outer end of the arm), a stimulus line will be said to have an orientation of $0 \mathrm{deg}$ at $12 \mathrm{o}$ 'clock, $30 \mathrm{deg}$ at $1 o^{\prime}$ clock, $60 \mathrm{deg}$ at $20^{\prime}$ clock, and so on.

(Received for publication June 25, 1973; accepted August 10, 1973.) 